\title{
Giant hemispheric multiloculated brain abscess
}

W en-ke Liu, Lu Ma, Bo-yong M ao

Department of Neurosurgery, West China Hospital, Sichuan University, Chengdu, Sichuan Province, P.R. China

A two-year-old baby was reffered to pediatric clinic for decreased appetite, pharyngeal congestion and lethargy. The pediatrician made a diagnosis of pharyngitis. Vital signs were normal and neurological examination revealed decreased muscle tone in the left sided limbs and right ptosis. The left pupil diameter was $2 \mathrm{~mm}$ whereas it was $5 \mathrm{~mm}$ on the right side. The white blood cell count was $8.04 \times 10^{9} / \mathrm{L}$ with differential counts of $67 \%$ neutrophils. Magnetic resonance imaging (MRI) revealed a giant cerebral hemispheric multiloculated lesion, which was hypointense on T1-weighted image and hyperintense on T2 [Figure 1].

The preoperative diagnosis was cystic glioma or other illdefined diseases. During the operation, a thick-walled giant multiloculated abscess containing thick pus was seen. The pus grew Staphylococcus aureus. Pathological examination also confirmed brain abscess [Figure 2a]. Patient recovered well without any neurological defect post-operatively. Follow-up computed tomography (CT) scan done at three months revealed no residual abscess [Figure 2b].

Differentiation between multiloculated brain abscess and cystic brain tumors such as low-grade glioma is often difficult with conventional and diffusion MRI. ${ }^{[1]}$ In our patient the brain abscess was possibly of otogenic

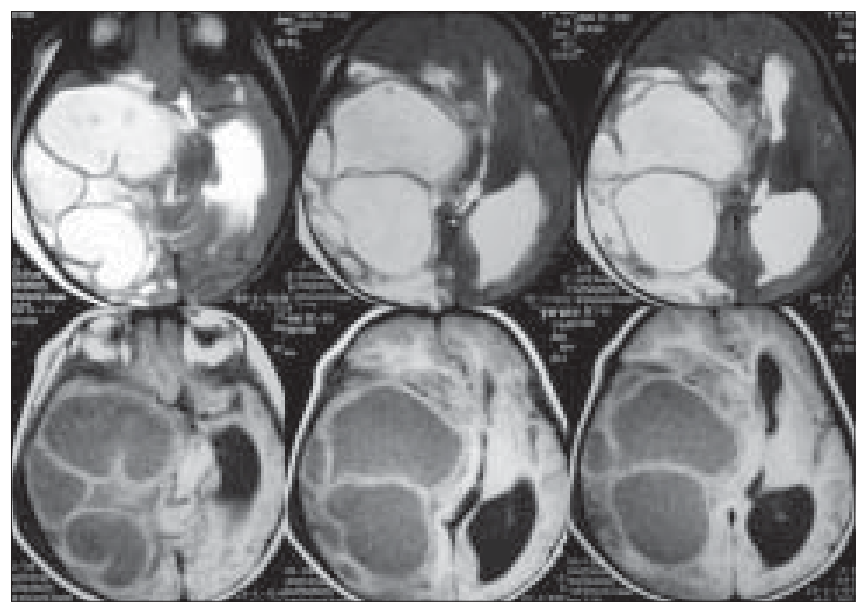

Figure 1: MRI revealing a huge multiloculated heterogeneous lesion of right hemisphere on $\mathrm{T} 1$ and $\mathrm{T} 2$ weighted images origin as the child had pharyngeal inection, in the past which could easily lead to tympanitis and hemispheric brain abscess. This is because the short and wide auditory tube in a child can get easily infected.

Surgical treatment should be attempted for reducing the mass effect, obtaining the pathologic diagnosis, and identifying infecting pathogens for facilitating selection of antibiotics. ${ }^{[2]}$ Excision seemed to be the most appropriate surgical choice for multiloculated abscess as recommended by Loftus et al. ${ }^{[3]}$

\section{Address for correspondence: Dr. Boyong Mao, \\ Department of Neurosurgery, West China Hospital, Sichuan University, Chengdu, Sichuan province, 610 041, P.R. China E-mail: liuwenke781103@yahoo.com.cn}

DOI: $10.4103 / 0028-3886.48795$

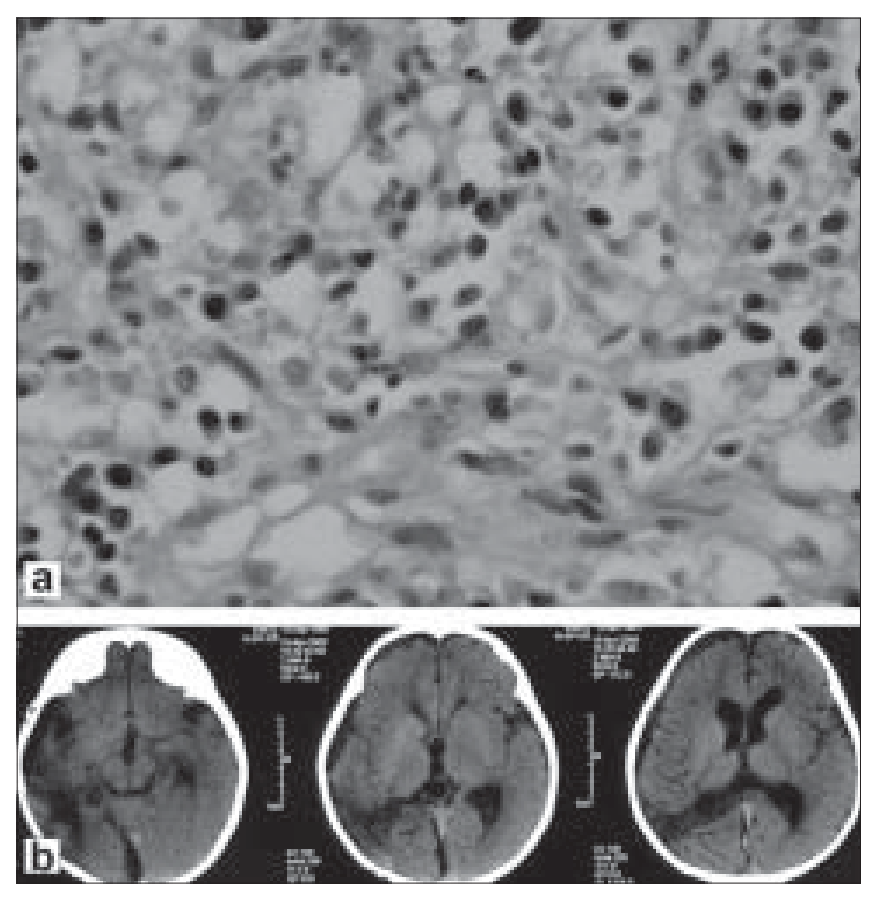

Figure 2: (a) Multiloculated brain abscess with lots of plasmocytes and neutrophils, (H and $E, \times 400)(b) C T$ scan revealing no recurrence of abscess for three months after operation 


\section{References}

1. Erdogan C, Hakyemez B, Yildirim N, Parlak M. Brain abscess and cystic brain tumor, discrimination with dynamic susceptibility contrast perfusion-weighted MRI. J Comput Assist Tomogr 2005;29:663-7.

2. Kratimenos G, Crockard HA. Multiple brain abscess: A review of fourteen cases. Br J Neurosurg 1991;5:153-61.

3. Loftus CM, Osenbach RK, Biller J. Diagnosis and management of brain abscess. In: Wilkins RH, Rengachary SS, editors. Neurosurgery. New York: McGraw-Hill Press, 1996. p. 3285-98.

Accepted on 26-01-2009

Source of Support: Nil, Conflict of Interest: None declared. 